\title{
Synaptic Proteins in the Postmortem Anterior Cingulate Cortex in Schizophrenia: Relationship to Treatment and Treatment Response
}

\author{
KA Barksdale', AC Lahti' and Rosalinda C Roberts*,', \\ 'Department of Psychiatry and Behavioral Neurobiology, University of Alabama, Birmingham, AL, USA
}

\begin{abstract}
The anterior cingulate cortex (ACC) is one of several brain regions that are abnormal in schizophrenia (SZ). Here we compared markers of synapse and mitochondrial function using western blots of postmortem ACC in: I) normal controls (NCs, $n=13$ ) vs subjects with SZ $(n=25)$; NC, treatment-resistant SZ, and treatment-responsive SZ; and 3) NC and SZ treated with typical or atypical antipsychotic drugs (APDs). Protein levels of synaptophysin, mitofusin-2, vGLUTI, and calcineurin did not differ between the NC and SZ group as a whole, or the NCs vs the SZ group divided by treatment response or type of APDs. In several cases, the levels of vGLUTI were minuscule or absent. The proportion of NCs lacking vGLUTI was significantly less than that of the SZ groups. There were several positive correlations across all subjects between: I) synaptophysin and VGLUTI; 2) synaptophysin and calcineurin; 3) synaptophysin and mitofusin; and 4) calcineurin and mitofusin. Synaptophysin and calcineurin were positively correlated in responders, and this correlation was significantly stronger than that in treatment-resistant SZ subjects or in NCs. Synaptophysin and calcineurin were positively correlated in SZ patients on atypical APDs; this correlation was significantly stronger than that in SZ patients on typical APDs or in NCs. Mitofusin-2 and calcineurin were positively correlated in SZ patients on atypical APDs and in NCs; this correlation was stronger in SZ patients on atypical rather than typical APDs or in NCs. The correlation between these proteins, which have roles in synaptic vesicle cycling, glutamate transmission, mitochondrial fusion, and calcium buffering, is complex and was differentially regulated among the groups.

Neuropsychopharmacology (2014) 39, 2095-2103; doi:I0.1038/npp.20I4.57; published online 2 April 20I4
\end{abstract}

\section{INTRODUCTION}

Schizophrenia (SZ) is a severe mental illness that manifests itself with psychosis, negative symptoms, and cognitive deficits. Antipsychotic drugs (APDs) improve psychotic symptoms, but approximately one third of the patients do not respond to treatment (Meltzer, 1997; Conley and Kelly, 2001). The reason for treatment resistance is poorly understood but appears to have a biological basis (Sheitman and Lieberman, 1998; Altamura et al, 2005) as a relationship between pathophysiology and the degree of treatment response has been shown in several neuroimaging studies (Rodríguez et al, 1997; Staal et al, 2001). In particular, the anterior cingulate cortex (ACC) has abnormalities linked to and predictive of treatment response (Lahti et al, 2009; Egerton et al, 2012). SPECT shows differential values for cerebral perfusion, an index of neuronal activity (Gemmell et al, 1990), in treatment-responsive $v s$-resistant SZ subjects (Rodríguez et al, 1997). APD treatment decreases regional cerebral blood flow ( $\mathrm{rCBF}$ ) in the ACC when

\footnotetext{
*Correspondence: Dr RC Roberts, Department of Psychiatry and Behavioral Neurobiology, University of Alabama, Sparks Center 865D, 1720, 7th Avenue South, Birmingham, AL 35294, USA, Tel: + I 205996 9373, E-mail: rcusidor@uab.edu

Received 20 December 2013; revised I4 February 20।4; accepted 4

March 20I4; accepted article preview online 7 March 2014
}

patients respond to treatment (Lahti et al, 2006). As rCBF is an index of synaptic activity, these studies suggest more synaptic activity in the ACC in SZ and a decrease with successful treatment. Consistent with this are MRS spectroscopy studies showing higher glutamate activity in the ACC in first episode or in never-medicated SZ and normalization with successful treatment (Theberge et al, 2003; Kegeles et al, 2010). Taken together, these studies implicate errors in synaptic activity and/or neurotransmission in the ACC in SZ, particularly at glutamatergic synapses.

Postmortem studies of the ACC in SZ indicate numerous abnormalities (Todtenkopf et al, 2005; Oni-Orisan et al, 2008; for review see Fornito et al, 2009; Kraguljac et al, 2012a,b). Neurobiological differences between treatment response and treatment resistance in SZ are rarely studied in postmortem tissue, but provide a strategy for trying to link psychosis with particular neuropathology (Roberts et al, 2012). In the present postmortem study we sought to examine several markers of synaptic function that may elucidate the underlying mechanisms of these deficits. We selected synaptophysin, vGLUT1, calcineurin, and Mitofusin-2 because they are informative about different but related aspects of normal synaptic function (Figure 1a).

Synaptophysin is a synaptic vesicle protein (Wiedenmann and Franke, 1985) with multiple functions including regulating activity-dependent bulk endocytosis (Kwon and 
a

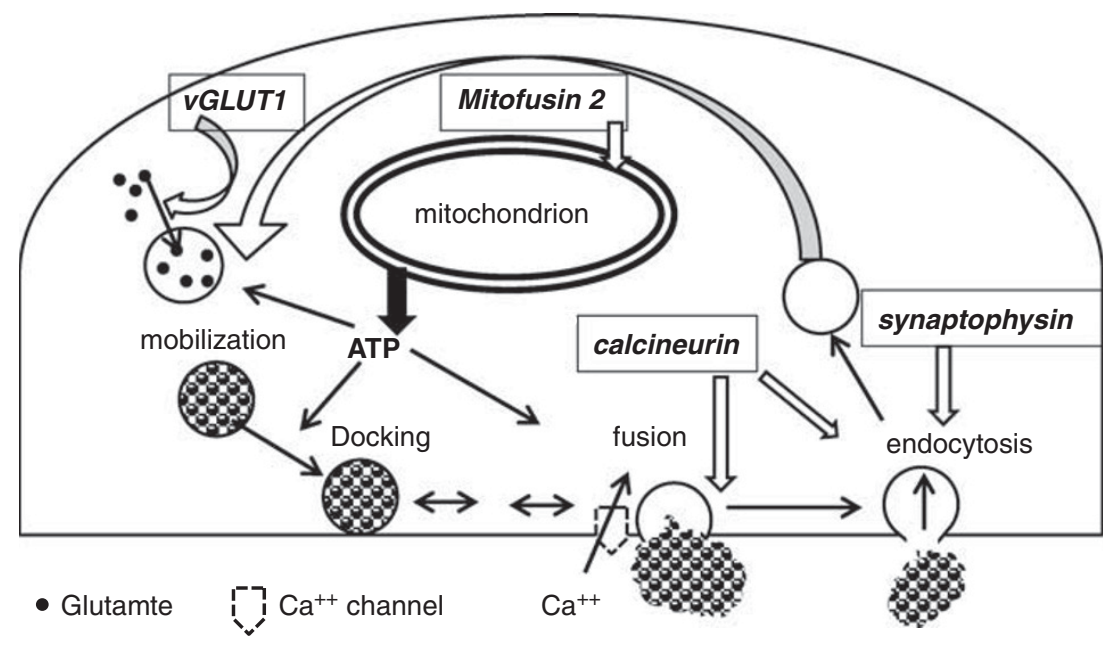

b

\begin{tabular}{|l|l|l|l|l|l|l|l|}
\hline & $\#$ & \multicolumn{1}{|c|}{ Age, yrs } & \multicolumn{1}{c|}{ PMI, hrs } & \multicolumn{1}{c|}{$\mathrm{pH}$} & Yrs frozen & Onset age & duration \\
\hline NC & 13 & $37.4 \pm 10.3$ & $14.0 \pm 6.5$ & $6.51 \pm 0.16$ & $10.4 \pm 2.6$ & na & na \\
\hline SZ & 25 & $42.5 \pm 11.6$ & $14.5 \pm 8.3$ & $6.65 \pm 0.32$ & $14.4 \pm 3.2$ & $22.1 \pm 4.8$ & $19.4 \pm 8.8$ \\
\hline t-test/M-W & & 0.191 & 0.858 & 0.266 & $\mathbf{0 . 0 0 0}$ & na & na \\
\hline TR+ & 12 & $40.3 \pm 9.7$ & $14.6 \pm 6.4$ & $6.63 \pm 0.38$ & $15.2 \pm 3.5$ & $20.6 \pm 4.9$ & $20.9 \pm 7.9$ \\
\hline Resp & 13 & $44.5 \pm 13.2$ & $14.4 \pm 10.1$ & $6.65 \pm 0.28$ & $13.5 \pm 2.6$ & $23.9 \pm 4.3$ & $17.6 \pm 10.2$ \\
\hline ANOVA/t-test & & 0.283 & 0.982 & 0.538 & $\mathbf{0 . 0 0 1}$ & 0.158 & 0.451 \\
\hline aAPD & 12 & $40.5 \pm 8.6$ & $15.2 \pm 9.6$ & $6.66 \pm 0.37$ & $14.1 \pm 2.9$ & $22.4 \pm 5.3$ & $19.2 \pm 8.7$ \\
\hline tAPD & 12 & $44.9 \pm 15.1$ & $13.6 \pm 7.9$ & $6.62 \pm 0.31$ & $15.0 \pm 3.4$ & $21.6 \pm 4.2$ & $19.9 \pm 9.8$ \\
\hline ANOVA/t-test & & 0.291 & 0.892 & 0.570 & $\mathbf{0 . 0 0 1}$ & 0.792 & 0.880 \\
\hline
\end{tabular}

\section{C}

\begin{tabular}{|l|c|l|l|l|l|l|l|c|c|}
\hline & $\#$ & race & sex & smoking & EtOH & suicide & DSM-IV & \#TR/resp & a/tAPD \\
\hline $\mathrm{NC}$ & 13 & $6 \mathrm{~W} / 7 \mathrm{~B}$ & $10 \mathrm{M} / 3 \mathrm{~F}$ & $5 \mathrm{Y} / 5 \mathrm{~N}$ & $2 \mathrm{Y} / 10 \mathrm{~N}$ & na & na & na & na \\
\hline $\mathrm{SZ}$ & 25 & $14 \mathrm{~W} / 11 \mathrm{~B}$ & $21 \mathrm{M} / 4 \mathrm{~F}$ & $15 \mathrm{Y} / 1 \mathrm{~N}$ & $9 \mathrm{Y} / 15 \mathrm{~N}$ & $6 \mathrm{Y} / 17 \mathrm{~N}$ & $12 \mathrm{U} / 12 \mathrm{P}$ & $\mathrm{na}$ & $\mathrm{na}$ \\
\hline$\chi^{2}$ & & 0.564 & 0.593 & $\mathbf{0 . 0 1}$ & 0.201 & $\mathrm{na}$ & $\mathrm{na}$ & $\mathrm{na}$ & $\mathrm{na}$ \\
\hline $\mathrm{TR}+$ & 12 & $5 \mathrm{~W} / 7 \mathrm{~B}$ & $9 \mathrm{M} / 3 \mathrm{~F}$ & $8 \mathrm{Y} / 0 \mathrm{~N}$ & $5 \mathrm{Y} / 6 \mathrm{~N}$ & $3 \mathrm{Y} / 8 \mathrm{~N}$ & $6 \mathrm{U} / 6 \mathrm{P}$ & $\mathrm{na}$ & $7 / 4$ \\
\hline Resp & 13 & $9 \mathrm{~W} / 4 \mathrm{~B}$ & $12 \mathrm{M} / 1 \mathrm{~F}$ & $7 \mathrm{Y} / 1 \mathrm{~N}$ & $4 \mathrm{Y} / 9 \mathrm{~N}$ & $3 \mathrm{Y} / 9 \mathrm{~N}$ & $6 \mathrm{U} / 6 \mathrm{P}$ & $\mathrm{na}$ & $5 / 7$ \\
\hline$\chi^{2}$ & & 0.327 & 0.466 & $\mathbf{0 . 0 3 0}$ & 0.326 & $\mathrm{~ns}$ & $\mathrm{~ns}$ & $\mathrm{na}$ & $\mathrm{na}$ \\
\hline aAPD & 12 & $7 \mathrm{~W} / 5 \mathrm{~B}$ & $10 \mathrm{M} / 2 \mathrm{~F}$ & $8 \mathrm{Y} / 1 \mathrm{~N}$ & $4 \mathrm{Y} / 8 \mathrm{~N}$ & $3 \mathrm{Y} / 7 \mathrm{~N}$ & $6 \mathrm{U} / 5 \mathrm{P}$ & $7 / 5$ & $\mathrm{na}$ \\
\hline tAPD & 11 & $6 \mathrm{~W} / 5 \mathrm{~B}$ & $9 \mathrm{M} / 2 \mathrm{~F}$ & $5 \mathrm{Y} / 0 \mathrm{~N}$ & $3 \mathrm{Y} / 7 \mathrm{~N}$ & $2 \mathrm{Y} / 9 \mathrm{~N}$ & $5 \mathrm{U} / 6 \mathrm{P}$ & $4 / 7$ & $\mathrm{na}$ \\
\hline$\chi^{2}$ & & 0.822 & 0.914 & $\mathbf{0 . 0 5 2}$ & 0.623 & $\mathrm{~ns}$ & $\mathrm{~ns}$ & $\mathrm{na}$ & $\mathrm{na}$ \\
\hline
\end{tabular}

Figure I (a) Diagram of a glutamatergic axon terminal showing several key components involved in vesicle cycling. vGLUTI inserts glutamate into synaptic vesicles. Calcineurin is necessary for fusion of the vesicles at the synapse and endocytosis. Synaptophysin is necessary for endocytosis. Mitofusin-2 is located in the mitochondria. Mitochondria produce ATP, which is necessary for all of these functions. (b, c) Demographic and other information; mean and standard deviation. Results of appropriate statistical tests are shown. APDs, antipsychotic medication; t, typical APDs; a, atypical APDs; ARS, age, race, sex; $\mathrm{EtOH}$, alcohol dependence; na, not applicable; P, chronic paranoid; PMI, postmortem interval; U, chronic undifferentiated type; unk, unknown.

Chapman, 2011). It is widely used as a marker of axon terminals (Calhoun et al, 1996). The vesicular glutamate transporters (vGLUTs) package glutamate into synaptic vesicles (Bellocchio et al, 2000). The amount of vGLUT corresponds to the amount of glutamate release; thus, increased protein levels will indicate more glutamate release at the synapse (Wojcik et al, 2004; Wilson et al, 2005).

Calcineurin is a major calcium/calmodulin-binding protein in the brain that regulates a number of neuronal functions, including synaptic transmission and plasticity (Baumgärtel and Mansuy, 2012). Presynaptically, calcineurin regulates elements of the synaptic vesicle cycle including recycling vesicle pool size (Sun et al, 2010) and bulk synaptic vesicle endocytosis during periods of intense synaptic activity (Clayton et al, 2009). Postsynaptically, calcineurin activity has been implicated in downstream regulation of dopaminergic signal transduction and in NMDA receptor-dependent synaptic plasticity (Greengard, 2001). Genetic analysis of the calcineurin pathway has identified potential susceptibility candidates in SZ (Gerber et al, 2003; Yamada et al, 2007).

Mitochondria, the source of $95 \%$ of the ATP in the brain, are involved in many brain disorders including SZ (Anglin et al, 2012; Manji et al, 2012). Mitochondria function as a dynamic network constantly undergoing fusion and fission, the balance of which is important in maintaining their structural integrity and function (Legros et al, 2002). Mitofusin-2 is a mitochondrial membrane protein that has 
a role in mitochondrial fusion and contributes to the maintenance and operation of the mitochondrial network (Bach et al, 2003). In addition, mitofusin-2 is necessary for transport of mitochondria and proper localization qin neuronal processes (Misko et al, 2010; Sheng and Cai, 2012).

The purpose of the present study was to compare protein levels for synaptophsyin, mitofusin, vGLUT1, and calcineurin in controls and SZ subjects as a combined cohort and divided by treatment response or type of treatment. We hypothesize that, in comparison to controls, SZ subjects who were treatment-resistant would have more profound alterations than treatment-responsive SZ subjects.

\section{MATERIALS AND METHODS}

\section{Postmortem Brain}

Human brains were obtained from the Maryland Brain Collection. All cases were obtained with consent from the next of kin with IRB approved protocols. The tissue was collected from adult SZ subjects $(n=25)$ and controls (NCs) $(n=13)$ from the rostral anterior cingulate gyrus. Demographics are shown in Figure $1 b$ and $c$.

Diagnosis was based on chart review, autopsy reports, medical records, and interviews with family. Postmortem cases were characterized using treatment-resistant criteria as we have done in our previous postmortem studies (Roberts et al, 2012). Briefly, treatment resistance is rated according to the criteria of Conley and Kelly (2001), which are a modification of the Kane criteria (Kane et al, 1988) and are defined as: 1) presence of a drug-refractory condition, which is defined as at least two treatment periods of adequate duration and dose with no clinical improvement; 2) persistence of illness for at least 5 years with no period of good social or occupational functioning; and 3) persistent positive psychotic symptoms.

\section{Western Blot Analyses}

Western blot assays were performed according to our previously reported methods (Perez-Costas et al, 2012), with the following exceptions. Initial analyses were performed to determine the optimal antibody concentrations and incubation times. The following antibodies were used: rabbit anti-vGLUT1, 1:10 000, Mab technologies (VGT1-3; Stone Mountain, GA, USA); mouse anti-calcineurin, 1:2000, BD Biosciences (610260; San Jose, CA, USA); rabbit antimitofusin-2, 1:1000, Sigma-Aldrich (M6444; St. Louis, MO, USA); mouse anti-synaptophysin, 1:5000, Chemicon/Millipore (MAB368; Temecula, CA, USA); and mouse anti-actin, 1:40 000, Millipore (MAB1501; Billerica, MA, USA). To confirm antibody specificity, vGLUT peptide (1:2500) was preincubated with the antibody for 1 hour, prior to blotting; this resulted in total elimination of the signal (Supplementary Figure 1A).

Approximately $50 \mathrm{mg}$ of frozen tissue was removed from the human ACC and placed directly into $2 \mathrm{ml}$ of lysis buffer at $4{ }^{\circ} \mathrm{C}$. Each sample was sonicated for $5 \mathrm{~s}$, and incubated at $4{ }^{\circ} \mathrm{C}$ for $15 \mathrm{~min}$ in a solution of $20 \mathrm{mM}$ Tris ( $\mathrm{pH} \mathrm{7.5),} 0.01 \%$ Triton X-100, $150 \mathrm{mM} \mathrm{NaCl}, 2 \mathrm{mM}$ EDTA, 2 mM EGTA, and a protease/phosphatase inhibitor cocktail (Sigma P8340).
The homogenate was centrifuged at $20800 \mathrm{~g}$ for $10 \mathrm{~min}$. The supernatant was recovered and protein concentrations were determined by the Lowry method (Bio-Rad 5000113, 500-0114).

Protein extracts $(60 \mu \mathrm{g})$ were loaded onto $4-20 \%$ gradient polyacrylamide gels along with a molecular weight marker (Bio-Rad). Proteins were resolved at $120 \mathrm{~V}$ for 1 hour, and then transferred at $30 \mathrm{~V}$ overnight onto PVDF membranes (Bio-Rad) at $4{ }^{\circ} \mathrm{C}$. The membranes were blocked and then antibody presence was detected by incubating the primary antibody with the PVDF membrane overnight at $4{ }^{\circ} \mathrm{C}$, with the exception of mitofusin-2, which was incubated for $72 \mathrm{~h}$ at $4{ }^{\circ} \mathrm{C}$. Next, the membranes were rinsed in TBST, incubated with the secondary antibody, and again rinsed in TBST. The bands were visualized using chemiluminescence (Bio-Rad; 170-5018), exposing GeneMate Blue Basic Autoradiography films. Membranes were blotted, stripped, and reblotted sequentially for vGLUT1, calcineurin, mitofusin-2, synaptophysin, and actin. Films were scanned at 600 dpi using a flatbed scanner, and optical density was measured using Image J-64 freeware (NIH).

\section{Data Analyses}

The individual performing the western blots and measuring the optical density was blinded to the identity of the groups (KB). Each gel contained a mixture of NCs and SZ subjects divided by response and type of APDs. Samples were run on duplicate gels and loaded in different orders to minimize potential artifacts. All values were first normalized to actin. Subsequently, each normalized protein was normalized to controls for each protein. These values were then averaged for the duplicate samples. The data were assessed for normality with a Kolmogorov-Smirnov test and then followed by parametric or nonparametric analysis as indicated. Chi-squared tests were performed for categorical measures. For demographic, tissue quality, or clinical characteristics that were significantly different between groups, a correlation analysis was performed to rule out potential confounds. The relationship between the proteins was analyzed by a correlation analysis across all subjects and then divided by diagnosis, treatment response, or the APD used. Subsequently, two correlation coefficients analyses were performed with VassarStats to determine betweengroup differences. Chi-Square analysis with Graph-Pad was performed on the difference in proportion of subjects in each group where vGLUT1 was absent.

\section{RESULTS}

Demographic analyses (Figure $1 b$ and $c$ ) indicated that there were no significant differences between groups with the exception of smoking and storage years. Correlation analyses between synaptophysin, vGLUT1, mitofusin-2, and calcineurin, and smoking and freezer time in each group showed no significant correlations (data not shown).

All proteins were detected at their correct molecular weight (Figure 2a). Protein levels of synaptophysin, vGLUT1, mitofusin-2, and calcineurin were not significantly different between the NC and SZ groups (Figure 2b).

Examples of western blots are shown for the NCs and the SZ group divided by treatment response or resistance 
a
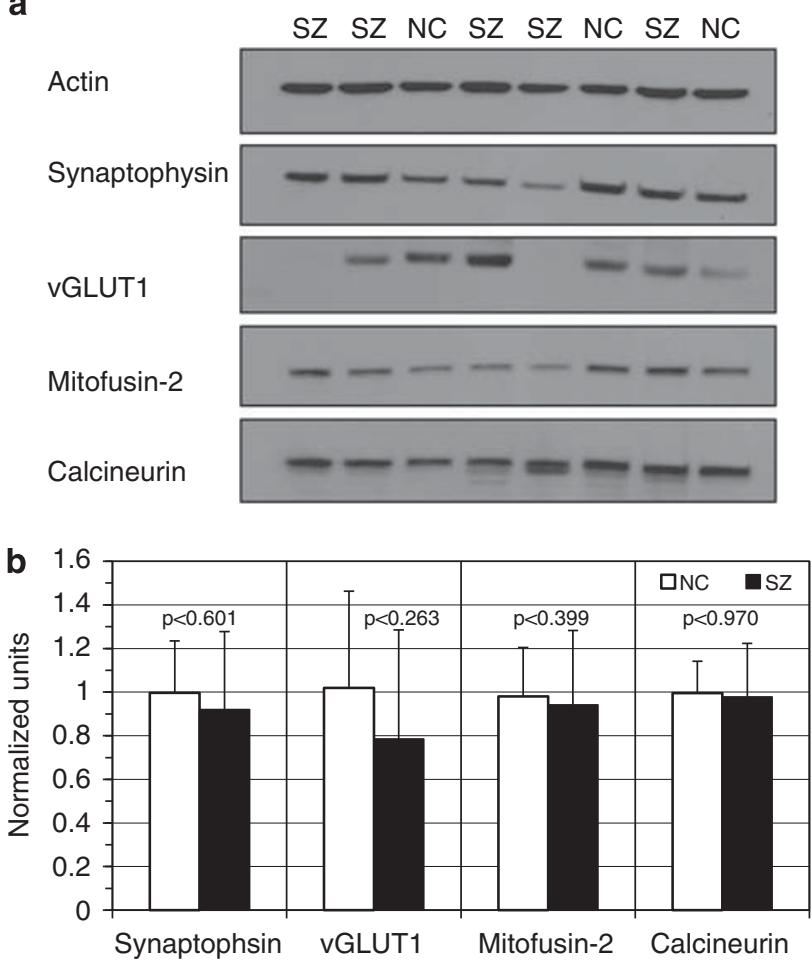

Figure 2 Protein levels for normal controls (NC) vs the combined Schizophrenia(SZ) cohort. All graphs show protein levels normalized to actin. (a) Representative western blots of one gel showing eight different subjects. Note that the vGLUTI signal completely disappears from two of the SZ cases and is markedly diminished in one NC case. (b) There were no statistically significant differences in any of the measures between groups: $p$ values are indicated.

(Figure 3a). Protein levels of synaptophysin, vGLUT1, mitofusin-2, and calcineurin were not significantly different between the NCs and SZ cases divided by treatment response (Figure $3 \mathrm{~b}$ ) or by type of treatment (Figure 3c).

In several samples, the levels of vGLUTt1 were minuscule $(<5 \%$ of average) or absent (see Figures $2 a$ and $3 a)$. This pattern was not observed for any of the other proteins. During preliminary experiments, to optimize antibody concentration and incubations times, vGLUT1 was always absent in these same individuals, meaning that the number of times this was replicated was greater than the two duplicate gels run for the final analysis. There was no biological reason to exclude these cases from the analyses as the protein levels for actin were normal. The proportion of cases with greater than $95 \%$ loss of vGLUT1 was compared across groups (Figure $3 \mathrm{~d}-\mathrm{f}$ ). The proportion of NCs lacking vGlut1 $(1 / 13,8 \%)$ was significantly lower than that of the combined SZ cohort $(5 / 25,20 \%)$ and all the subgroups of SZ. Moreover, the TR-SZ group had a higher percentage of subjects with no vGLUT1 than the responders; subjects on tAPDs had a higher percentage of subjects with no vGLUT1 than the subjects on aAPDs. There were no apparent characteristics that might explain why these cases lacked vGLUT1 (Supplementary Figure 1B). In addition, correlation analysis between vGLUT1 and PMI, years frozen or nicotine did not reveal significant correlations in NCs, SZ subjects and any of the subgroups of SZ (Supplementary Figure 1C-E).

There were positive correlations between synaptophysin and each of the other three proteins across all subjects (Figure $4 \mathrm{a}-\mathrm{c}$ ). In addition, calcineurin and mitofusin were positively correlated (Figure $4 \mathrm{~d}$ ). When separating the NC and SZ groups, significant correlations for mitofusin with synaptophysin or calcineurin were present in both groups. The correlations for synaptophysin with vGLUT1 and with calcineurin remained significant only in SZ. However, the difference in correlations between NC and SZ subjects was not significant.

Next, we examined protein correlations in the groups based on treatment response/resistance or on the type of APD. Synaptophysin and calcineurin were positively correlated in responders, but not in NCs or TR-SZ cases (Figure $5 a-c$ ). The degree of correlation between the responders and both the TR-SZ subjects and NCs was significantly different. Synaptophysin and calcineurin were positively correlated in the SZ cases treated with atypical APDs, and this correlation was significantly different from that of SZ subjects treated with typical APDs and the NCs (Figure 5d-f). Synaptophysin and mitofusin were all positively correlated in the NCs and in both treatment groups, with no differences between groups (data not shown). Calcineurin and mitofusin were positively correlated in NCs and in the SZ patients treated with atypical APDs (Figure 5g-i). This correlation was significantly different between the SZ patients treated with atypical APDs and the SZ patients on typical APDs and the NC groups.

\section{DISCUSSION}

Although levels of the proteins we studied were not abnormal in the combined cohort of SZ subjects or in the subgroups, some interesting results emerged. vGLUT1 was absent or vastly reduced in several cases, and there were no distinguishing features that could explain these findings. The proportion of the population in each subset of SZ with cases devoid of vGLUT1 was greater than in controls, signifying a possible propensity in the SZ population for a loss or vast reduction of vGLUT1. The only association of treatment resistance with vGLUT1 levels was that a greater proportion of the TR population lacked vGLUT1 than did responders, whereas average protein levels between groups were similar. MRS spectroscopy in the ACC showed increased glutamate in SZ patients who were still symptomatic after treatment, compared with that in SZ patients who were responsive after treatment (Egerton et al, 2012), suggesting that reducing glutamate transmission is related to successful treatment. One mechanism to decrease glutamate transmission would be to reduce vGLUT1 levels. However, our data show that the responders had similar overall levels of vGLUT1 as those of the TR-SZ subjects, suggesting an alternative or additional mechanism for reducing glutamate in the treatment responders.

A previous report indicated a decrease in vGLUT1 (OniOrisan et al, 2008) in the ACC in elderly SZ subjects. Our results support overall decreases in vGLUT1, but the data did not achieve significance because of the large variance 
a

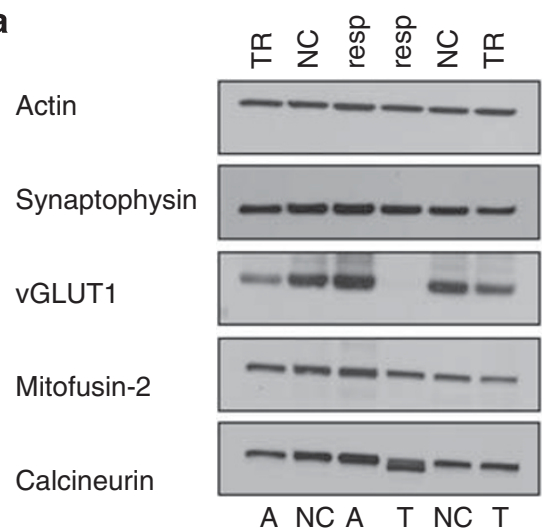

b

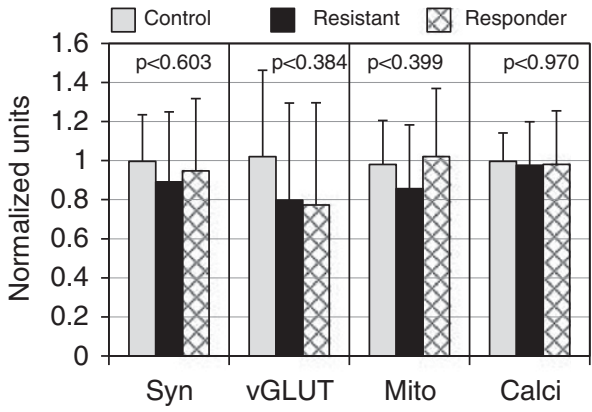

c

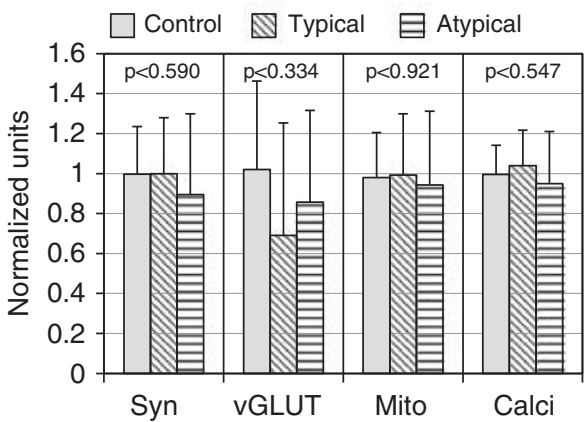

d

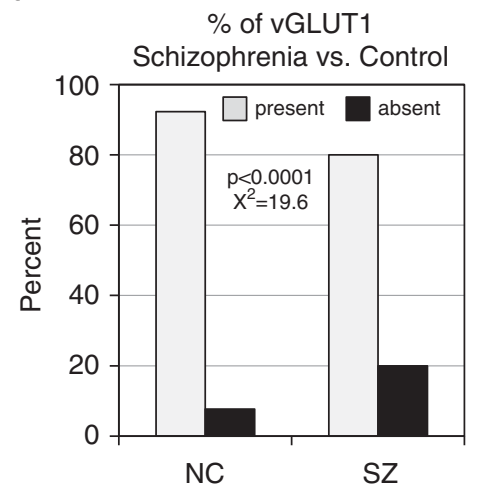

$\mathbf{e}$

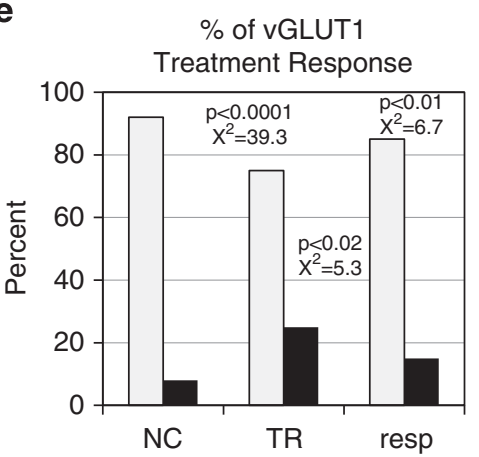

f

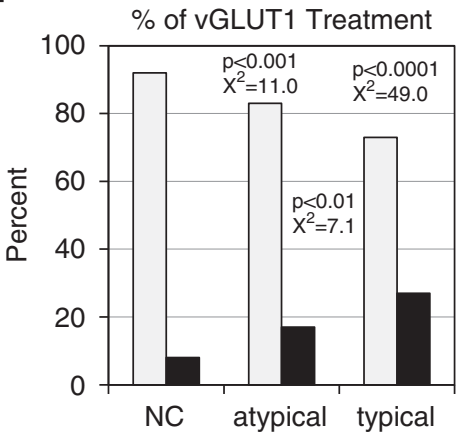

Figure 3 Protein levels for NCs vs subgroups of SZ cases divided by treatment response or treatment. All graphs show protein levels normalized to actin. (a) Representative western blots of one gel showing six different subjects. Treatment response (resp) or resistance (TR) is indicated above the blots. The type of APD used (atypical, A, or typical, T) is indicated below the blots. (b, c) There were no statistically significant differences in any of the measures between groups divided by treatment response (b) or type of APD treatment (c). (d-f) Graphs showing the percentage of cases of each group that showed the absence ( $<95 \%$ loss) or presence of vGLUTI. p values and $\chi^{2}$ values ( I degree of freedom and a two-tailed test) are shown above subgroups, indicating a difference between that subgroup and controls, or between subgroups, indicating differences between those subgroups. (d) Subjects with SZ had a higher proportion of subjects lacking vGLUTI than did NCs. (e) Both responder (resp) and resistant (TR) cases had higher proportions of cases lacking vGLUT I than did NCs. The TR group also had a higher proportion of cases lacking VGLUTI than did the responders. (f) Both SZ groups had higher proportions of cases lacking vGLUTI than did NCs. The group on typical APDs also had a higher proportion of cases lacking vGLUTI than did the SZ cases on atypical APDs.

due to the total dropout of vGLUT from several of our samples. This discrepancy may be due to several factors. Our cohort was younger, racially mixed, and the tissue sample area may have come from different areas of the ACC than those used in the study of Oni-Orisan et al (2008). Glutamatergic terminals from cortex or thalamus contain vGLUT1 and vGLUT2, respectively (Fremeau et al, 2004), and are used to label the respective connections. Recent evidence shows that the ACC, at least the rostral part where we sampled, is involved in the default mode network, a series of cortical connections mediating cerebral activity at rest (Greicius et al, 2003). This network is impaired in SZ (Garrity et al, 2007) with abnormalities in vGLUTs.

Synaptophysin is widely used as a marker of axon terminals (Calhoun et al, 1996). Although it is not necessary for neurotransmitter release (McMahon et al, 1996), synaptophysin has an important role in regulating synaptic vesicle endocytosis (Daly et al, 2000). Our results are consistent with previous findings showing no changes in synaptophysin mRNA or protein in the ACC in SZ 

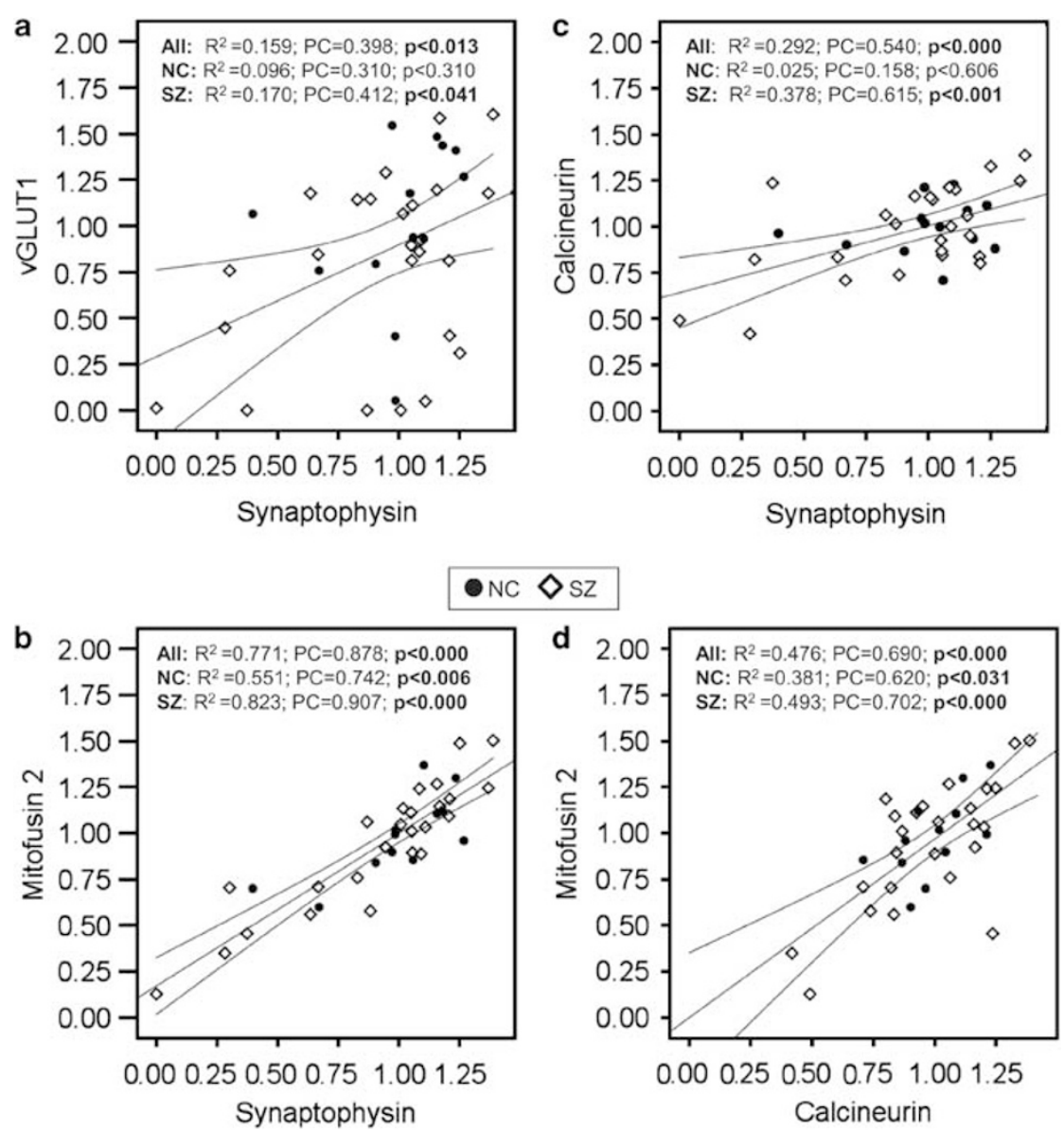

Figure 4 Graphs of correlation analyses of proteins that had significant interactions across all subjects. (a) synaptophysin and vGLUTI, (b) synaptophysin and Mitofusin-2, (c) synaptophysin and calcineurin, and (d) calcineurin and mitofusin-2. $R^{2}$, Pearson's correlation (PC), and the $p$ values are shown in each graph for all subjects combined, and then subdivided by diagnosis (NC and SZ). A two-coefficient correlation analysis indicated that the NC and SZ cases did not differ significantly for any of these correlations.

(Eastwood and Harrison, 2001). These results imply normal synaptic density, which is surprising because the reduced neuropil hypothesis of SZ (Selemon and Goldman-Rakic, 1999) posits fewer cortical synaptic connections.

Calcineurin is active in regulating synaptic vesicle endocytosis, and dopaminergic and glutamate signal transduction. Genetic studies have linked genes in the calcineurin pathway to SZ (Yamada et al, 2007). The present study is the first to report calcineurin protein levels in the ACC in SZ, and they were normal. The prefrontal cortex and hippocampus also do not show any changes in this protein (Kozlovsky et al, 2006). However, calcineurin appears to be differentially affected, depending on the brain region, as an increased ratio of calcineurin neurons has been reported in the caudate nucleus in SZ (Wada et al, 2012).

Pathology in mitochondria has long been implicated in $\mathrm{SZ}$, as shown by genetic, proteomic, enzymatic, and anatomical abnormalities (for reviews see, Anglin et al, 2012; Manji et al, 2012). The present study is the first to report mitofusin-2 protein levels in postmortem SZ cases, and they were normal. Normal protein levels suggest that mitochondrial fusion and maintenance and operation of the mitochondrial network may be intact. However, although mitofusin-2 may be normal, there are many other mitochondrial proteins involved in fusion and fission that may be abnormal.

No changes in protein levels suggest that vGLUt1, synaptophysin, calcineurin, and mitfusin-2 are not altered in the ACC in SZ or involved in treatment response. However, all our subjects were treated with APDs, which could confound our results. APDs administered to animals do not affect synaptophysin (Lidow et al, 2001) or vGLULT1 (Oni-Orisan et al, 2008). To our knowledge, there are no reports on the effects of APDs on mitofusn-2. However, APDs reduce RNA and protein levels of the alpha subunit of calcineurin (Rushlow et al, 2005) and may have normalized high levels of calcineurin, masking an abnormality in SZ.

Across all subjects, we found strong positive correlations between mitofusin-2 and both synaptophysin and calcineurin. The high correlation of mitofusin-2 with synaptophysin and calcineurin suggests a tight interaction between mitofusin-2 and these two proteins. This makes sense because mitofusin-2 is a marker of mitochondria that are producing ATP, which is critical for all cellular processes. The data suggest that the relationship between healthy mitochondria and both synaptophysin and calcineurin is present in controls and is intact in SZ. 
a
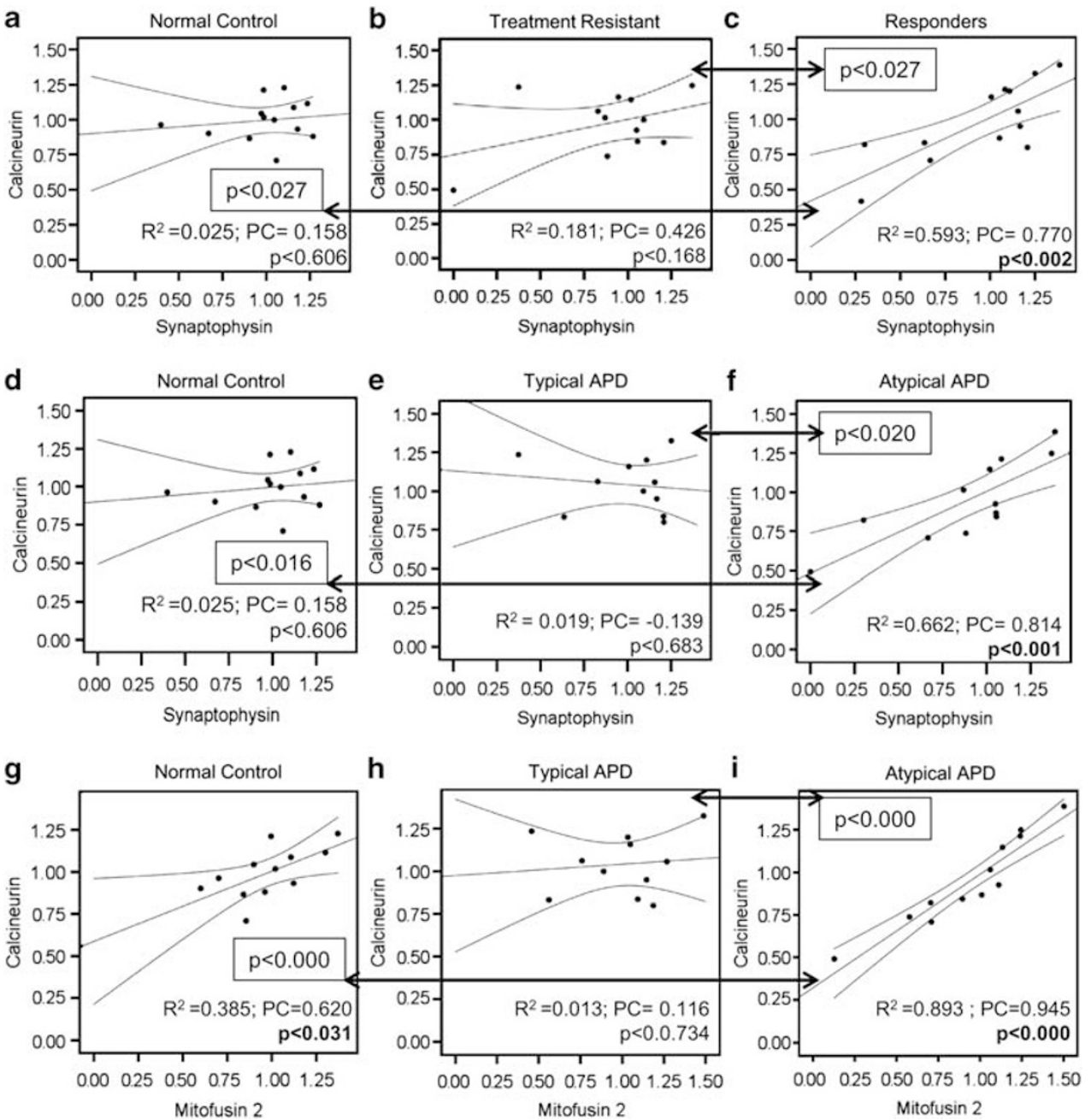

Figure 5 Graphs of correlation analyses of proteins that had significant differences between controls and SZ cases divided by treatment response or type of treatment. $R^{2}$, Pearson's correlation (PC), and the $p$ values are shown in each graph. The results of two coefficient correlation analysis between groups are shown in boxes with arrows connecting the groups with significant differences. (a-c) Graphs of correlations between synaptophysin and calcineurin in groups divided by treatment response. A significant correlation existed only in the responders, which was significantly different from that of controls and TR-SZ subjects. A one tail $t$-test was used for $(a-c)$ because our hypothesis was that responders would be more similar to controls. ( $d-f)$ Graphs of correlations between synaptophysin and calcineurin in each group divided by type of treatment. A significant correlation existed only in the SZ cases treated with atypical APDs, which was significantly different from that in the controls and in the SZ cases treated with typical APDs. (g-i) Graphs of correlations between Mitofusin-2 and calcineurin in each group divided by type of treatment. A significant correlation existed in the NCs and in the SZ subjects treated with atypical APDs. The correlation for the atypical APD group was significantly different from NCs and from the SZ subjects treated with typical APDs. (a) and (d) are identical and are replicated for comparative purposes. Two-tailed t-tests were used for (d-i).

Across all subjects, there were significant positive correlations between synaptophysin and both vGLULT1 and calcineurin. The correlation between synaptophysin and vGLUT1 was expected, given that they are integral parts of or are linked to synaptic vesicles in glutamate synapses. Similarly, the correlation between synaptophysin and calcineurin was also expected due to their common function in endocytosis. When dividing the subjects by diagnosis, the relationships were no longer present in the NCs, but were present in the SZ patients. This observation was surprising and may indicate a compensatory mechanism in the interaction between the proteins in SZ.

The correlation between synaptophysin and calcineurin was present in treatment responders, but not in TR-SZ cases or NCs. As TR cases and NCs did not have a correlation, the data suggest that a mechanism that gets these two proteins to act in concert may be a compensatory mechanism related to treatment response. The positive correlation just in the responders is probably not driven by the type of APD because the TR and responder subgroups were comprised of equal numbers of subjects on typical $v s$ atypical APDs.

We found a differential effect of the type of APD on some of the protein correlations. Calcineurin was positively correlated with both synaptophysin and mitofusin-2 only in SZ patients on atypical APDs. This suggests that atypical APDs may affect the relationship of these proteins, whereas typical APDs have no effect. Moreover, atypical APDs may cause a compensatory response in the interaction between the proteins. Thus, the positive correlation of calcineurin with both mitofusin-2 and synaptophysin in the 
SZ group as a whole may be driven by the subjects on atypical APDs.

In summary, all protein levels that we studied were normal in SZ and were not differentially affected by treatment or treatment response status. However, our findings do not preclude posttranslational modifications. A positive correlation between calcineurin and synaptophysin was linked to treatment response and also to treatment with atypical APD, although perhaps by independent mechanisms. The positive correlation between mitofusin-2 and calcineurin may be related to treatment with atypical APDs as well. Future studies should include a drug-free group of SZ cases. Protein-protein interactions may also reveal the molecular network that is enhanced or abnormally present in SZ.

\section{FUNDING AND DISCLOSURE}

This research was supported by NIH grants MH081014 (ACL), and a 5K12GM088010-02 MERIT Scholarship (KAB). The authors declare no conflict of interest.

\section{ACKNOWLEDGEMENTS}

We thank the Maryland Brain Collection for the brain samples (especially Dr Robert Conley, diagnoses, and Dr Andree Lessard, dissections), and Dr Miguel MelendezFerro and Dr Emma Perez-Costas for technical expertise with the western blots.

\section{REFERENCES}

Altamura AC, Bassetti R, Cattaneo E, Vismara S (2005). Some biological correlates of drug resistance in schizophrenia: a multidimensional approach. World J Biol Psychiatry 6(Suppl 2): 23-30.

Anglin RE, Mazurek MF, Tarnopolsky MA, Rosebush PI (2012). The mitochondrial genome and psychiatric illness. Am J Med Genet B Neuropsychiatr Genet 159B: 749-759.

Bach D, Pich S, Soriano FX, Vega N, Baumgartner B, Oriola J et al (2003). Mitofusin-2 determines mitochondrial network architecture and mitochondrial metabolism. A novel regulatory mechanism altered in obesity. J Biol Chem 278: 17190-17197.

Baumgärtel K, Mansuy IM (2012). Neural functions of calcineurin in synaptic plasticity and memory. Learn Mem 19: 375-384.

Bellocchio EE, Reimer RJ, Fremeau RT Jr, Edwards RH (2000). Uptake of glutamate into synaptic vesicles by an inorganic phosphate transporter. Science 289: 957-960.

Calhoun ME, Jucker M, Martin LJ, Thinakaran G, Price DL, Mouton PR (1996). Comparative evaluation of synaptophysinbased methods for quantification of synapses. J Neurocytol 12: 821-828.

Clayton EL, Anggono V, Smillie KJ, Chau N, Robinson PJ, Cousin MA (2009). The phospho-dependent dynamin-syndapin interaction triggers activity-dependent bulk endocytosis of synaptic vesicles. J Neurosci 29: 7706-7717.

Conley RR, Kelly DL (2001). Management of treatment resistance in schizophrenia. Biol Psychiatry 50: 898-911.

Daly C, Sugimori M, Moreira JE, Ziff EB, Llinás R (2000). Synaptophysin regulates clathrin-independent endocytosis of synaptic vesicles. Proc Natl Acad Sci 97: 6120-6125.

Eastwood SL, Harrison PJ (2001). Synaptic pathology in the anterior cingulate cortex in schizophrenia and mood disorders.
A review and a western blot study of synaptophysin, GAP-43 and the complexins. Brain Res Bull 55: 569-578.

Egerton A, Brugger S, Raffin M, Barker GJ, Lythgoe DJ, McGuire PK et al (2012). Anterior cingulate glutamate levels related to clinical status following treatment in first-episode schizophrenia. Neuropsychopharmacology 37: 2515-2521.

Fornito A, Yücel M, Dean B, Wood SJ, Pantelis C (2009). Anatomical abnormalities of the anterior cingulate cortex in schizophrenia: bridging the gap between neuroimaging and neuropathology. Schizophr Bull 35: 973-993.

Fremeau RT Jr, Voglmaier S, Seal RP, Edwards RH (2004). VGLUTs define subsets of excitatory neurons and suggest novel roles for glutamate. Trends Neurosci 27: 98-103.

Garrity AG, Pearlson GD, McKiernan K, Lloyd D, Kiehl KA, Calhoun VD (2007). Aberrant 'default mode' functional connectivity in schizophrenia. Am J Psychiatry 164: 450-457.

Gemmell HG, Evans NT, Besson JA, Roeda D, Davidson J, Dodd MG et al (1990). Regional cerebral blood flow imaging: a quantitative comparison of technetium-99m-HMPAO SPECT with C15O2 PET. J Nucl Med 10: 1595-1600.

Gerber DJ, Hall D, Miyakawa T, Demars S, Gogos JA, Karayiorgou $\mathrm{M}$ et al (2003). Evidence for association of schizophrenia with genetic variation in the $8 \mathrm{p} 21.3$ gene, PPP3CC, encoding the calcineurin gamma subunit. Proc Natl Acad Sci USA 100: 8993-8998.

Greicius MD, Krasnow B, Reiss AL, Menon V (2003). Functional connectivity in the resting brain: a network analysis of the default mode hypothesis. Proc Natl Acad Sci USA 100: 253-258.

Greengard P (2001). The neurobiology of dopamine signaling. Biosci Rep 21: 247-269.

Kane JM, Honigfeld G, Singer J, Meltzer H (1988). Clozapine in treatment-resistant schizophrenics. Psychopharmacol Bull 24: 62-67.

Kegeles LS, Abi-Dargham A, Frankle WG, Gil R, Cooper TB, Slifstein $\mathrm{M}$ et al (2010). Increased synaptic dopamine function in associative regions of the striatum in schizophrenia. Arch Gen Psychiatry 67: 231-239.

Kozlovsky N, Scarr E, Dean B, Agam G (2006). Postmortem brain calcineurin protein levels in schizophrenia patients are not different from controls. Schizophr Res 83: 173-177.

Kraguljac NV, Reid MA, White D, Jones R, den Hollander J, Lowman D et al (2012a). Neurometabolites in schizophrenia and bipolar disorder - a systematic review and metaanalysis. Psychiatry Res 203: 111-125.

Kraguljac NV, Reid MA, White DM, den Hollander J, Lahti AC (2012b). Regional decoupling of N-acetyl-aspartate and glutamate in schizophrenia. Neuropsychopharmacology 37: 2635-2642.

Kwon SE, Chapman ER (2011). Synaptophysin regulates the kinetics of synaptic vesicle endocytosis in central neurons. Neuron 70: 847-854.

Lahti AC, Weiler MA, Holcomb HH, Tamminga CA, Carpenter WT, McMahon R (2006). Correlations between rCBF and symptoms in two independent cohorts of drug-free patients with schizophrenia. Neuropsychopharmacology 31: 221-230.

Lahti AC, Weiler MA, Holcomb HH, Tamminga CA, Cropsey KL (2009). Modulation of limbic circuitry predicts treatment response to antipsychotic medication: a functional imaging study in schizophrenia. Neuropsychopharmacology 34: 2675-2690.

Legros F, Lombès A, Frachon P, Rojo M (2002). Mitochondrial fusion in human cells is efficient, requires the inner membrane potential, and is mediated by mitofusins. Mol Biol Cell 12: 4343-4354.

Lidow MS, Song ZM, Castner SA, Allen PB, Greengard P, GoldmanRakic PS (2001). Antipsychotic treatment induces alterations in dendrite- and spine-associated proteins in dopamine-rich areas of the primate cerebral cortex. Biol Psychiatry 49: 1-12.

Manji H, Kato T, Di Prospero NA, Ness S, Beal MF, Krams M et al (2012). Impaired mitochondrial function in psychiatric disorders. Nat Rev Neurosci 13: 293-307. 
McMahon HT, Bolshakov VY, Janz R, Hammer RE, Siegelbaum SA, Südhof TC (1996). Synaptophysin, a major synaptic vesicle protein, is not essential for neurotransmitter release. Proc Natl Acad Sci USA 93: 4760-4764.

Meltzer HY (1997). Treatment-resistant schizophrenia-the role of clozapine. Curr Med Res Opin 14: 1-20 Review.

Misko A, Jiang S, Wegorzewska I, Milbrandt J, Baloh RH (2010). Mitofusin 2 is necessary for transport of axonal mitochondria and interacts with the Miro/Milton complex. J Neurosci 30: 4232-4240.

Oni-Orisan A, Kristiansen LV, Haroutunian V, Meador-Woodruff $\mathrm{JH}$, McCullumsmith RE (2008). Altered vesicular glutamate transporter expression in the anterior cingulate cortex in schizophrenia. Biol Psychiatry 63: 766-775.

Perez-Costas E, Melendez-Ferro M, Rice MW, Conley RR, Roberts RC (2012). Dopamine pathology in schizophrenia: analysis of total and phosphorylated tyrosine hydroxylase in the substantia nigra. Front Psychiatry 3: 31.

Reid MA, Stoeckel LE, White DM, Avsar KB, Bolding MS, Akella NS et al (2010). Assessments of function and biochemistry of the anterior cingulate cortex in schizophrenia. Biol Psychiatry 68: 625-633.

Roberts R, Roche J, Somerville S, Conley R (2012). Ultrastructural distinctions between treatment responders and non-responders in schizophrenia: postmortem studies of the striatum. In: L'Abate L (eds) Mental Illness/Book 2. InTech: Rijeka, Croatia, pp 261-286.

Rodríguez VM, Andrée RM, Castejón MJ, Zamora ML, Alvaro PC, Delgado JL et al (1997). Fronto-striato-thalamic perfusion and clozapine response in treatment-refractory schizophrenic patients. A 99mTc-HMPAO study. Psychiatry Res 76: 51-61.

Rushlow WJ, Seah YH, Belliveau DJ, Rajakumar N (2005). Changes in calcineurin expression induced in the rat brain by the administration of antipsychotics. J Neurochem 4: 587-596.

Selemon LD, Goldman-Rakic PS (1999). The reduced neuropil hypothesis: a circuit based model of schizophrenia. Biol Psychiatry 45: 17-25.

Sheitman BB, Lieberman JA (1998). The natural history and pathophysiology of treatment resistant schizophrenia. J Psychiatr Res 32: 143-150.
Sheng ZH, Cai Q (2012). Mitochondrial transport in neurons: impact on synaptic homeostasis and neurodegeneration. Nat Rev Neurosci 13: 77-93.

Staal WG, Hulshoff Pol HE, Schnack HG, van Haren NE, Seifert N, Kahn RS (2001). Structural brain abnormalities in chronic schizophrenia at the extremes of the outcome spectrum. Am J Psychiatry 158: 1140-1142.

Sun T, Wu XS, Xu J, McNeil BD, Pang ZP, Yang W et al (2010). The role of calcium/calmodulin-activated calcineurin in rapid and slow endocytosis at central synapses. J Neurosci 30: 11838-11847.

Theberge J, Al-Semaan Y, Williamson PC, Menon RS, Neufeld RW, Rajakumar N et al (2003). Glutamate and glutamine in the anterior cingulate and thalamus of medicated patients with chronic schizophrenia and healthy comparison subjects measured with 4.0-T proton MRS. Am J Psychiatry 160: 2231-2233.

Todtenkopf MS, Vincent SL, Benes FM (2005). A cross-study metaanalysis and three-dimensional comparison of cell counting in the anterior cingulate cortex of schizophrenic and bipolar brain. Schizophr Res 73: 79-89.

Wada A, Kunii Y, Ikemoto K, Yang Q, Hino M, Matsumoto J et al (2012). Increased ratio of calcineurin immunoreactive neurons in the caudate nucleus of patients with schizophrenia. Prog Neuropsychopharmacol Biol Psychiatry 37: 8-14.

Wiedenmann B, Franke WW (1985). Identification and localization of synaptophysin, an integral membrane glycoprotein of $\mathrm{Mr}$ 38000 characteristic of presynaptic vesicles. Cell 41: 1017-1028.

Wilson NR, Kang J, Hueske EV, Leung T, Varoqui H, Murnick JG et al (2005). Presynaptic regulation of quantal size by the vesicular glutamate transporter VGLUT1. J Neurosci 25: 6221-6234.

Wojcik SM, Rhee JS, Herzog E, Sigler A, Jahn R, Takamori S et al (2004). An essential role for vesicular glutamate transporter 1 (VGLUT1) in postnatal development and control of quantal size. Proc Natl Acad Sci USA 101: 7158-7163.

Yamada K, Gerber DJ, Iwayama Y, Ohnishi T, Ohba H, Toyota T et al (2007). Genetic analysis of the calcineurin pathway identifies members of the EGR gene family, specifically EGR3, as potential susceptibility candidates in schizophrenia. Proc Natl Acad Sci USA 104: 2815-2820.

Supplementary Information accompanies the paper on the Neuropsychopharmacology website (http://www.nature.com/npp) 